\title{
Salienz unter Einheimischen und Zugezogenen \\ Ein empirischer Vergleich
}

\author{
Cornelia Lorenz (Paderborn)
}

\begin{abstract}
The local background may affect the salience of regiolectal variants. In my paper I am going to investigate this influence: I have tested, whether the same regiolectal variants attract varying attention of locals and newcomers in Eastwestfalia, where the variants are spread autochthonally. In Eastwestfalia, an important precondition was fulfilled; natives aged between 20 and 30 years, who have been linguistically socialized in Eastwestfalia, usually have an active regiolectal competence but no dialect competence. Depending on the local background, interesting trends were observed: The longer the duration of the stay, decreasing salience was detected in some cases and increasing salience in others. The different reasons for this surprising development will be explained in my paper.
\end{abstract}

\section{$1 \quad$ Problemstellung}

Woran es liegt, dass linguistische Laien bestimmte sprachliche Merkmale als auffälliger empfinden als andere, ist bisher nicht abschließend geklärt (cf. Elmentaler/Gessinger/Wirrer 2010: 114). Eine Kategorisierung der Merkmale nach linguistischen Kriterien in primär und sekundär (cf. Schirmunski 1928/1929: 166) hat sich ohne die Berücksichtigung sprachlicher Attitüden nicht bewährt (cf. Mihm 2000: 2109). Die Wahrnehmung und Einordnung der sprachlichen Merkmale durch linguistische Laien lässt sich nicht allein anhand phonetischphonologischer Messungen prognostizieren (cf. Hundt 2010: 181). ${ }^{1}$ Vielmehr unterscheidet sich die Wahrnehmung nach Lenz (2010: 94) individuell. Bei ihrer Argumentation greift Lenz - in Anlehnung an Trudgill (1986: 11) - auf den Begriff Salienz zurück:

Unter Salienz wird hier die kognitive Auffälligkeit eines sprachlichen Merkmals verstanden, in dem Sinne, dass ein sprachliches Element aus seinem Kontext hervorgehoben wird und dadurch dem Sprachbewusstsein leichter und schneller zugänglich ist als nicht-saliente Varianten (Lenz 2010: 94)

Als ein salienzverstärkender Faktor wird die regionale Herkunft diskutiert: Elmentaler, Gessinger und Wirrer (2010:117) erwähnen diese als möglichen Einflussfaktor, Purschke (2011: 73, 85) hat diesen Faktor bereits in seiner Forschung berücksichtigt. Hinskens, Auer und Kerswill (2004: 45) werfen sogar die Frage auf, ob die Salienz sprachlicher Merkmale generell anhand von einheimischen oder auswärtigen Sprechern getestet werden sollte. Hier setzt die vorliegende Untersuchung an. Sie vergleicht, inwiefern Einheimische und Zugezogene autochthone Regionalismen als auffällig wahrnehmen. Im Folgenden werden zunächst Konzeption und Ablauf der empirischen Untersuchung vorgestellt und Erwartungen formuliert. Anschließend werden die Ergebnisse dargestellt und erläutert.

\footnotetext{
${ }^{1}$ Eine detaillierte Diskussion dieser Problematik nehmen Lenz (2010) und Purschke (2011) vor.

Linguistik online 66, 4/14 - http://dx.doi.org/10.13092/1o.66.1576 


\section{Salienztests}

Die vorliegende empirische Studie prüft die Salienz solcher phonetisch-phonologischer Varianten, die Einheimische mehrheitlich als wenig oder gar nicht auffällig wahrnehmen. Zu diesem Zweck wurde sie in einer ausgesprochen dialektschwachen Region durchgeführt: In Ostwestfalen (einer Region im niederdeutschen Raum) ist der Abbau der Basisdialekte so weit fortgeschritten, dass der Großteil der jungen Erwachsenen über keinerlei Dialektkenntnisse verfügt (cf. Wirrer 1998: 310-311; Stellmacher 1987: 12, 27; Möller 2008: $16,65-66)^{2}$

Insgesamt wurden 145 Personen an einem einzigen Studienort befragt, und zwar im ostwestfälischen Bielefeld. Die Probanden wurden in drei Gruppen eingeteilt, die im Rahmen der Auswertung miteinander verglichen wurden, nämlich einheimische Ostwestfalen, Zugezogene aus dem niederdeutschen Raum und Zugezogene aus dem mittel- und oberdeutschen Raum. Es wurden nur solche einheimische und zugezogene Probanden bei der Auswertung berücksichtigt, auf die die folgenden Auswahlkriterien zutrafen: Alle einheimischen Probanden waren in Ostwestfalen aufgewachsen und hatten den Großteil ihres Lebens dort verbracht; keiner dieser Probanden hatte Ostwestfalen länger als vier Jahre lang verlassen. Die zugezogenen Probanden waren frühestens mit 16 Jahren nach Ostwestfalen gezogen und hatten zum Zeitpunkt der Datenaufnahme seit mindestens einem und maximal acht Jahren in Ostwestfalen gelebt. Personen, die aus anderen Regionen in Westfalen oder aus dem niederfränkischen Raum nach Ostwestfalen gezogen waren, wurden wegen der großen dialektalen und regiolektalen Ähnlichkeit dieser Regionen mit Ostwestfalen ausgeschlossen. Alle befragten Personen waren nicht jünger als 20 und nicht älter als 30 Jahre. Die Probanden selbst sowie mindestens ein Elternteil waren mit Deutsch als einziger Muttersprache aufgewachsen.

Nach Ausschluss der Personen, auf die mindestens ein Auswahlkriterium nicht zutraf, umfasste die Auswertung die Daten von 101 Personen. Die Probanden verteilten sich folgendermaßen auf die drei Untersuchungsgruppen: 32 Zugezogene aus dem niederdeutschen, nicht-westfälischen Raum, 32 Zugezogene aus dem mittel- und oberdeutschen Raum und 37 einheimische Ostwestfalen (cf. Nemeth 2011: 101; Lorenz i. V.).

Im Vorfeld der Erhebung wurden 20 Sätze aufgezeichnet, die eine Reihe von regiolektalen Merkmalen aufwiesen. Die Konzeption des Salienztests orientiert sich am Forschungsprojekt "Sprachvariation in Norddeutschland" (SIN). ${ }^{3}$ Durch Beobachtungen und Befragungen unter Studierenden sowie Literaturrecherchen (König 1989a, 1989b; Lauf 1996) wurden Varianten ermittelt, die von jungen Erwachsenen in Ostwestfalen verwendet werden (cf. Nemeth 2011: 102-103; Lorenz i. V). Zu den Varianten wurden Stimuli ausgewählt und in Sätze eingebettet: Die g-Spirantisierung im Auslaut z. B. wurde in zwei verschiedenen Sätzen durch die Stimuli lie[ç]t 'liegt' und Rückwe[ç] 'Rückweg' abgefragt.

Eine in Ostwestfalen beheimatete Sprecherin sprach die 20 Sätze im Umfang von jeweils drei bis sechs Wörtern als Tonaufnahme ein. Diese Tonaufnahmen wurden den Probanden über Kopfhörer vorgespielt. Die Sätze wiesen phonetisch-phonologische, syntaktische oder phraseologische Regionalismen auf, die in Ostwestfalen verbreitet sind. Um ein Priming für

\footnotetext{
2 Für solche Teile des niederdeutschen Raums schließen Herrgen und Schmidt eine Anwendung des Regionalsprachbegriffs weitgehend aus (cf. Schmidt/Herrgen 2011: 75), stattdessen verwenden sie den Begriff Regiolekt (cf. ibd.: 68). Im Folgenden werden die Sprechlagen zwischen Standardvarietät und Dialekten dementsprechend als Regiolekt und die im Regiolekt verbreiteten sprachlichen Merkmale als regiolektal bezeichnet.

${ }^{3}$ Eine Beschreibung des SIN-Projekts nehmen Elmentaler et al. (2006) vor; erste Ergebnisse des Salienztests stellen Elmentaler/Gessinger/Wirrer (2010) vor; eine Studie zu Salienz in Anlehnung an das SIN-Projekt präsentiert Hettler im vorliegenden Band.
} 
ostwestfälische Regionalismen zu vermeiden, wurden zusätzlich regiolektale Merkmale aus verschiedenen anderen, großlandschaftlichen Dialektregionen verwendet. Sämtliche Regionalismen hatten sich im Vorfeld der Untersuchung bei den Befragungen unter Studierenden und bei der Literaturrecherche (cf. König 1989a, 1989b; Lauf 1996) als regional gebunden erwiesen (cf. Nemeth 2011; Lorenz i. V.). Insgesamt wiesen die Sätze 32 Merkmale auf: 25 Merkmale waren phonetisch-phonologisch motiviert, sieben Merkmale phraseologisch und syntaktisch. Von den 25 phonetisch-phonologischen Merkmalen sind 14 in Ostwestfalen gebräuchlich (cf. ibd.), neun Merkmale sind in anderen Regionen verbreitet (cf. König 1989a, 1989b; Lauf 1996) und zwei Abweichungen waren als Versprecher nicht intendiert. Auf jeden der 20 Sätze entfielen null bis maximal drei Regionalismen.

Gerade die einheimischen, ortsfesten Probanden waren nicht daran gewöhnt, regiolektale Ostwestfalismen wahrzunehmen. Um den Schwierigkeitsgrad für die Einheimischen durch eine Begrenzung der Abspielvorgänge nicht zu erhöhen, hatten alle Probanden die Möglichkeit, sich die Tonaufnahmen beliebig oft anzuhören. Die Mehrzahl der Probanden hörte sich jeden Satz zwei- bis dreimal an. Nach dem Abspielen wurde jeder Proband mündlich nach Auffälligkeiten ${ }^{4}$ gefragt. Alle Angaben wurden von der Autorin protokolliert und aufgezeichnet. Viele Probanden nahmen dabei eine Einschätzung der Merkmale vor. Häufig bewerteten sie die Merkmale negativ; besonders die phraseologischen und syntaktischen Merkmale wurden von den Probanden weniger als regiolektale Merkmale, sondern vielmehr als Indizien für einen niedrigen Bildungsstand gedeutet (cf. Lorenz i. V.). Bislang wurden die Kommentare nicht ausgewertet. Es wurde lediglich untersucht, ob die Probanden die Merkmale als auffällig angaben oder nicht.

\section{Erwartungen}

Aus den Ergebnissen des vorgestellten Salienztests lässt sich ableiten, inwiefern sich die drei Untersuchungsgruppen (einheimische Ostwestfalen, Zugezogene aus dem niederdeutschen, nicht-westfälischem Raum und Zugezogene aus dem mittel- und oberdeutschen Raum) bei der Einordnung der Stimuli unterscheiden. Es ist zu erwarten, dass sich die Einheimischen vor allem von solchen Zugezogenen unterscheiden, die aus Regionen stammen, in denen das abgefragte Merkmal autochthon nicht verbreitet ist.

Außerdem lassen die Ergebnisse Schlüsse auf die kommunikative Verwendung der abgefragten Merkmale zu: Saliente Regionalismen können gezielt dazu eingesetzt werden, um Situationen als informell $\mathrm{zu}$ kennzeichnen und um die eigene regionale Zugehörigkeit zu betonen (cf. Salewski 1998: 28; Reershemius 2009: 145). Das lexikalisch in dat gebunden auftretende, unverschobene /t/ wird z. B. in mehreren großlandschaftlichen Dialektregionen dementsprechend verwendet (cf. Schmidt/Herrgen 2011: 172; Elmentaler/Gessinger/Wirrer 2010: 134), nämlich u. a. im Moselfränkischen (cf. Lenz 2003: 203). Saliente Merkmale können zudem in formellen Situationen unterdrückt werden: Die Ergebnisse eines Vorlesetests und eines Salienztests unter 14 Frauen aus Schleswig-Holstein (Elmentaler/Gessinger/Wirrer 2010: 120) lassen darauf schließen, dass hochsaliente Varianten beim Vorlesen tendenziell vermieden werden. Regionalismen mit geringer Salienz realisieren Einheimische dagegen eher unbewusst und weitgehend situationsunabhängig - und zwar häufig ohne sie in irgendeiner Weise als auffällig wahrzunehmen (cf. Elmentaler/Gessinger/Wirrer 2010: 117-118; Hundt 2010: 181). Welche der abgefragten Merkmale dazu geeignet sind, von Einheimischen und Zugezogenen als "regionale

\footnotetext{
4 Die Frage „was fällt auf?" wurde offen formuliert. Es wurde bewusst darauf verzichtet, die Schriftsprache als Orientierungsrahmen vorzugeben. Dadurch wurden die Probanden motiviert, nicht nur Merkmale zu nennen, die sie selbst als Abweichung von der Schriftsprache einordneten, sondern auch Merkmale, die ihnen ungewöhnlich erschienen, ohne dass sie diese Einschätzung bewusst begründen konnten (cf. Lorenz i. V.).
} 
Identitätsmarker" (Salewski 1998: 28) eingesetzt und erkannt zu werden, lässt sich also durch Salienztests ermitteln.

\section{$4 \quad$ Regionale Verbreitung von vier phonetisch-phonologischen Variablen}

Im Folgenden werden die Ergebnisse des Salienztests anhand von vier phonetischphonologischen Variablen vorgestellt. $\mathrm{Zu}$ jeder Variable wurde mindestens ein Stimulus für die in Ostwestfalen gebräuchliche Variante mittels Salienztest abgefragt. Anschließend wurde durch einen Vergleich der Testergebnisse festgestellt, wie stark sich die Ergebnisse der drei Untersuchungsgruppen unterscheiden.

\section{1 $\quad g$-Spirantisierung im Auslaut}

Der Plosiv /g/ im Auslaut wird in Ostwestfalen regiolektal nicht auslautverhärtet als [k] realisiert, sondern nach dunklen Vokalen als [x] und nach hellen Vokalen sowie Liquiden als [ç] spirantisiert. Ausschließlich beim Derivationssuffix $<$ ig $>$ entspricht die spirantisierte Realisierung der Aussprachenorm (cf. Krech 2009: 84); in anderen Kotexten gilt sie als regional markiert. Die $g$-Spirantisierung im Auslaut entspricht dem niederdeutschen Substrat (cf. Stellmacher 1977: 96, 2000: 175; Scheel 1963: 383). Als regiolektale Ausprägung ist sie weit über Ostwestfalen hinaus verbreitet, nämlich im gesamten niederdeutschen sowie im berlinischen und mitteldeutschen Raum. Im westmitteldeutschen und sächsischen Raum wird die Spirantisierung nach hellen Vokalen als / / / koronalisiert (cf. Elspaß/Möller 2003ff.: Runde 1, Frage 18 du kriegst, Runde 1, Frage 15a Tag, Runde 1, Frage 15b Zeug, Runde 2, Frage 22d weg, König 1989b: 302-306; Lauf 1996: 199; Mihm 2000: 2113). In der vorliegenden Untersuchung wurde die in Ostwestfalen gebräuchliche spirantisierte, nicht-koronalisierte Ausprägung mithilfe der beiden Stimuli lie [ç]t und Rückwe[ç] abgefragt, also ausschließlich unmittelbar nach Langvokal.

\subsection{Realisierung der Affrikate [pf] im Anlaut als Frikativ [f]}

Die Affrikate /pf/ wird nördlich der Speyerer Linie weder dialektal noch regiolektal realisiert: Aufgrund der nicht bzw. nicht vollständig durchgeführten Zweiten Lautverschiebung ist die Affrikate im niederdeutschen und mitteldeutschen Raum basisdialektal unbekannt und wird dialektal als unverschobenes /p/ realisiert (cf. König 2004: 63; Scheel 1963:384). Im Ostmitteldeutschen wird die Affrikate östlich der Pfund/Fund-Isoglosse anlautend dialektal als einfacher Frikativ gesprochen (cf. König 2004: 230). Regiolektal wird die Affrikate im gesamten niederdeutschen und mitteldeutschen Raum im Anlaut als Frikativ [f] realisiert (cf. König 1989a: 101, 1989b: 259; Lauf 1996: 199; Mihm 2000: 2113). Im gesamten niederdeutschen Raum wird also anstatt des dialektal unverschobenen /p/ regiolektal der Frikativ verwendet, der - im Gegensatz zur Affrikate /pf/ - dialektal im Niederdeutschen vorkommt. Eine solche Kontrastminderung zwischen Dialekt und Standardvarietät durch eine mittelbare Substratinterferenz bezeichnet Elmentaler (2005: 403) als "indirekte Lernerinterferenz".

Die regiolektale, indirekte Lernerinterferenz wurde durch den Stimulus [f]lanze 'Pflanze' abgefragt. Vergleichend wurde eine Variante mit realisierter Affrikate geprüft: unge[pf]legt 'ungepflegt'. Diese Ausprägung der Variable entspricht der Standardlautung und ist autochthon südlich der Speyerer Linie verbreitet (cf. Krech 2009: 80).

\subsection{Realisierung von [ar] als Langmonophthong [a:]}

Folgt das Phonem /r/ unmittelbar auf einen Vokal, wird /r/ in vielen Regionen im deutschen Sprachraum vokalisiert ausgesprochen oder getilgt (cf. König 1989a: 75-76). Die Allophone [r], [R] und [в] sowie die Vokalisierung von /r/ nach Vokal als [e], z. B. [Jtaek] 'stark' oder 
[hivf] 'Hirsch' entsprechen der Aussprachenorm (cf. Krech 2009: 87). In Ostwestfalen wird /r/ regiolektal so stark vokalisiert, dass die vokalisierte Ausprägung einem volltonigen [a] entspricht. Der Vokal, der vor dem vokalisierten /r/ steht, wird dabei gedehnt. Folgt also /r/ auf den Kurzvokal /a/ wird /r/ unter Ersatzdehnung des volltonigen Phonems /a/ getilgt. Die Lautfolge $<$ ar $>$ wird in Ostwestfalen als Langmonophthong [a:] realisiert, z. B. [Jta:k]. Eine solche Realisierung als Langmonophthong ist in Westfalen, im nordniederdeutschen Raum südlich der Aller sowie punktuell in den westmitteldeutschen Städten Wittlich und Mainz gebräuchlich (cf. Elspaß/Möller 2003ff.: Runde 1, Frage 16b Karte; König 1989b: 194). Im Salienztest wurde diese Realisierung durch die beiden Stimuli F[a:]be 'Farbe' und g[a:] 'gar' abgefragt.

\subsection{Sekundärdiphthongierung}

Geht /r/ ein anderer Kurzmonophthong als /a/ voran, wird dieser Vokal in der regiolektalen, in Ostwestfalen verbreiteten Ausprägung gedehnt und zudem auffällig geschlossen artikuliert. Das Phonem /r/ wird dann durch ein volltoniges [a] oder [ $\varepsilon$ ] ersetzt, z. B. [hi:af] oder [hi: $\left.\varepsilon \int\right]$. Bei dieser Realisierung entsteht also ein Diphthong. In Anlehnung an Scheel (1963: 382-383) und Lauf (1996: 202) wird dieser im Folgenden als Sekundärdiphthong und die regiolektale Ausprägung als Sekundärdiphthongierung bezeichnet. Die Sekundärdiphthongierung ist in Westfalen, im nordniederdeutschen Raum südlich der Aller sowie punktuell im mittelbairischen Regensburg verbreitet (cf. König 1989b: 206-209: Lauf 1996: 206, 209; Mihm 2000: 2114-2115). Die Variante wurde anhand des Stimulus W[o:a]t 'Wort' abgefragt.

\section{Ergebnisse}

Für jeden Probanden wurde ermittelt, wie viele Stimuli er für jede in Ostwestfalen verbreitete Variante als auffällig angab. Diese Anzahl wurde zur Gesamtzahl der in den Tonaufnahmen enthaltenen Stimuli pro Variante in Relation gesetzt. Gab der Proband also z. B. die Hälfte der vorkommenden Stimuli für eine Variante als auffällig an, lautete das Testergebnis, dass für den Probanden die Variante in 50 Prozent der Fälle salient war. Zum Vergleich von Probandengruppen wurden die individuellen Werte innerhalb der Gruppen jeweils gemittelt. Die in der Untersuchung festgestellten, gemittelten Werte unter den Einheimischen (37 Personen), Zugezogenen aus dem nicht-westfälischen, niederdeutschen Raum (32 Personen) und Zugezogenen aus dem mittel- und oberdeutschen Raum (32 Personen) werden im Folgenden miteinander verglichen. Zudem werden solche Probanden gesondert aufgeführt, die aus Regionen stammen, in denen die abgefragte Variante nicht verbreitet ist. Diese Personen kennen solche Varianten nicht aus ihrer Heimatregion, aber vielleicht bereits aus Ostwestfalen.

Die in Ostwestfalen verbreiteten Varianten von drei der vier Variablen wurden bereits von Elmentaler, Gessinger und Wirrer (2011: 116-119) im Rahmen eines Salienztests unter 48 Probandinnen im Alter von ca. 50 Jahren aus Schleswig-Holstein, Brandenburg-Berlin und dem Südniederrhein abgefragt, nämlich die g-Spirantisierung im Auslaut nach langen Vokalen, die Realisierung der Affrikate [pf] im Anlaut als Frikativ und die Realisierung von [ar] als Langmonophthong [a:]. Alle 48 Probanden lebten in Regionen, in denen die abgefragte Variante regiolektal verbreitet ist (cf. König 1989b: 206-209; Lauf 1996: 206, 209; Mihm 2000: 2114-2115), so dass ihnen die entsprechenden Varianten vertraut sein dürften. Die Untersuchungsgruppen lassen sich daher mit den einheimischen Ostwestfalen und den Zugezogenen aus dem Verbreitungsgebiet der drei Varianten vergleichen. Auch Hettler fragte zwei der vier Varianten unter 80 Probanden im Alter zwischen 18 und 85 Jahren aus Bremen und Hamburg in einem Salienztest ab (cf. Hettler 2014), nämlich die gSpirantisierung im Auslaut nach Langvokal, Liquid und Kurzvokal sowie die Realisierung der 
Affrikate [pf] im Anlaut als Frikativ. Auch in diesen beiden Städten ist jeweils die in Ostwestfalen verwendete Variante regiolektal verbreitet (cf. König 1989b: 206-209; Lauf 1996: 206, 209; Mihm 2000: 2114-2115). ${ }^{5}$

\section{1 $\quad g$-Spirantisierung im Auslaut}

In der vorliegenden Studie wurde die Auffälligkeit der $g$-Spirantisierung im Auslaut nach Langvokal anhand der Stimuli lie[c] $t$ und Rückwe[c] untersucht. Fast alle Einheimischen und Zugezogenen nahmen die beiden Varianten als auffällig wahr: Die einheimischen Ostwestfalen gaben die g-Spirantisierung in 89 Prozent aller Fälle im Salienztest an, die beiden Zugezogenengruppen in 97 Prozent der Fälle. Von den Zugezogenen stammen nur sechs Probanden aus Regionen im oberdeutschen Raum, in denen die $g$-Spirantisierung nicht verbreitet ist; diesen Probanden fielen jeweils beide Stimuli auf. Die $g$-Spirantisierung im Auslaut wies also sehr hohe Salienzwerte auf, die sich nur geringfügig zwischen den drei Untersuchungsgruppen unterscheiden. Demnach wirkte sich die regionale Herkunft bei dieser Variable allenfalls sehr schwach auf die ermittelten Salienzwerte aus.

Elmentaler, Gessinger und Wirrer (2010: 117) fragten die g-Spirantisierung nach Langvokal mit fünf Stimuli ab. Die Stimuli wurden von 50 bis 100 Prozent der Probanden erkannt. Die Autoren ordnen die Variante dementsprechend als hochsalient ein, bemerken aber einschränkend: "Auch hier gibt es wortbezogene und regionale Differenzen, die aber aufgrund der insgesamt hohen Salienz nicht sehr stark ins Gewicht fallen" (ibd.: 118). Bei Hettler dagegen bemerkten die durch den Stimulus $W e[c]$ ' 'Weg' abgefragte $g$-Spirantisierung im Auslaut nach Langvokal nur 43 Prozent der Probanden. Worauf diese vergleichsweise niedrige Salienz zurückzuführen ist, ist noch nicht geklärt (cf. Hettler 2014).

\subsection{Realisierung der Affrikate [pf] im Anlaut als Frikativ [f]}

Die Salienz der regiolektalen Ausprägung der Affrikate /pf/ im Anlaut wurde in der vorliegenden Studie mithilfe des Stimulus [f]lanze geprüft. Die in der Untersuchung festgestellten, gemittelten Werte der Salienz von [f]lanze betrugen 43 Prozent unter den einheimischen Ostwestfalen, 34 Prozent unter den Zugezogenen aus dem niederdeutschen Raum und 53 Prozent unter den Zugezogenen aus dem mittel- und oberdeutschen Raum. Unter den Probanden in der Untersuchung von Elmentaler, Gessinger und Wirrer (2010: 117-118) fällt die ermittelte Salienz noch wesentlich geringer aus: Nur ein einziger von 48 Probanden gab einen von zwei abgefragten Stimuli als auffällig an. In Hettlers Korpus erkennen immerhin 23 der 80 Gewährspersonen die Variante; das entspricht 29 Prozent der Gruppe (cf. Hettler 2014) und damit weitgehend den in der vorliegenden Untersuchung festgestellten Werten für die niederdeutschen Zugezogenen und einheimischen Ostwestfalen.

Im gesamten niederdeutschen und mitteldeutschen Raum wird die Affrikate /pf/ im Anlaut als einfacher Frikativ [f] realisiert (cf. König 1989a: 101, 1989b: 259; Lauf 1996: 199; Mihm 2000: 2113). In der vorliegenden Studie stammen nur sieben Probanden aus Regionen, in denen die Affrikate dialektal und regiolektal in allen Stellungen realisiert wird, nämlich aus dem oberdeutschen Raum und aus Ostfranken. Jedem dieser sieben Probanden fiel die Artikulation der Affrikate als Frikativ auf. Unter diesen Probanden erweist sich die Variante also als hochsalient.

Neben der regiolektalen Ausprägung wurde auch die der Aussprachenorm entsprechende Variante der Variable (cf. Krech 2009: 80) auf ihre Salienz überprüft: Die in der Untersuchung festgestellten, gemittelten Salienzwerte des Stimulus unge[pf]legt betrug 86

\footnotetext{
${ }^{5}$ Einige Merkmale wurden auch von Mihm (1985) untersucht. Da die Untersuchung von Mihm allerdings knapp 30 Jahre zurückliegt, werden die Ergebnisse hier nicht vergleichend herangezogen.
} 
Prozent unter einheimischen Ostwestfalen, 88 Prozent unter den Zugezogenen aus dem niederdeutschen Raum und 53 Prozent unter den Zugezogenen aus dem mittel- und oberdeutschen Raum. Von den sieben Probanden aus dem oberdeutschen und ostfränkischen Raum fielen einer einzigen Probandin beide Realisierungen der Affrikate - als [f] und [pf] auf. Von den 94 Probanden aus dem mittel- und oberdeutschen Raum gaben 31 beide Varianten und elf keine der beiden Varianten an. Der überwiegenden Anzahl der Probanden aus dem mittel- und oberdeutschen Raum fiel die standardsprachliche Affrikate auf. Die Zugezogenen, denen die regiolektale Ausprägung auffiel, stammten größtenteils aus dem oberdeutschen Raum.

Alle Probanden gaben mehrheitlich die Variante als auffällig an, die in ihrer Herkunftsregion autochthon nicht verbreitet ist. Die Probanden aus dem niederdeutschen und dem mitteldeutschen Raum orientierten sich nicht dahingehend an der standardlautenden Variante [pf] und die oberdeutschen Zugezogenen nicht an der in Ostwestfalen verbreiteten Variante [f], dass sie kontrastiv die Variante aus der eigenen Herkunftsregion als auffällig wahrgenommen hätten. Die hohe Anzahl derjenigen, die beide Varianten als auffällig angaben, zeigt allerdings eine Unsicherheit in der Zuordnung. Diese Unsicherheit könnte auf die Testsituation zurückzuführen sein: Die Probanden nehmen in einer Testsituation vermutlich nicht nur solche Varianten als auffällig wahr, die in ihrem Alltag nicht vorkommen, wie die Affrikate [pf] im Anlaut in Ostwestfalen. Vielmehr ist davon auszugehen, dass sie ihre Wahrnehmung mit der Schriftsprache vergleichen: Dass [pf] - im Gegensatz zu [f] - dem Graphem <pf $>$ entspricht, dürfte die Probanden dazu veranlasst haben, die ihnen vertraute Variante [f] aufgrund der Abweichung vom Graphem als auffällig einzustufen und dementsprechend im Test anzugeben.

\subsection{Realisierung von [ar] als Langmonophthong [a:]}

Die Salienz der regiolektalen Realisierung von $<$ ar $>$ als [a:] wurde anhand der beiden Stimuli $F[a:] b e$ und $g[a:]$ geprüft. Im absoluten Auslaut war die Variante lediglich für vier Prozent aller Probanden salient. Im Silbenauslaut dagegen erreichte sie einen deutlich höheren Salienzwert von 25 Prozent. Der Stimulus $g[a:]$ wurde nach $F[a:] b e$ abgespielt. Niemandem fiel ausschließlich $g[a:]$ auf: Die vier Probanden denen $g[a:]$ auffiel, hatten bereits $F[a:] b e$ als auffällig angegeben.

Unter den einheimischen Ostwestfalen betrug der in der Untersuchung festgestellte, gemittelte Salienzwert der beiden Varianten elf Prozent (g[a:]: 0 Prozent, F[a:]be: 22 Prozent). Unter den Zugezogenen aus dem niederdeutschen Raum wies der gemittelte Wert acht Prozent auf und lag damit leicht unterhalb des gemittelten Salienzwertes der einheimischen Ostwestfalen. Nach Ausschluss von sieben Personen, in deren Heimatregion das Merkmal autochthon verbreitet ist, betrug der gemittelte Salienzwert unter den Zugezogenen aus dem niederdeutschen Raum ebenfalls acht Prozent. Auch in Bezug auf dieses Merkmal fällt der gemessene Salienzwert in der Untersuchung von Elmentaler, Gessinger und Wirrer (2010: 117) noch niedriger aus: Eine von 48 Probanden gab den abgefragten Stimulus als auffällig an (das entspricht einem Anteil von nur 2,1 Prozent).

Unter den Zugezogenen aus dem mittel- und oberdeutschen Raum betrug der in der vorliegenden Studie festgestellte, gemittelte Salienzwert 27 Prozent und lag damit wesentlich höher als in den beiden anderen Gruppen. Niemand aus der Gruppe stammte aus der Umgebung von Wittlich und Mainz, wo die Variante punktuell verbreitet ist (cf. Elspaß/Möller 2003ff.: Runde 1, Frage 16b Karte; König 1989b: 194). Für knapp die Hälfte der Gruppe - 43 Prozent - war F [a:]be salient. Die in der Untersuchung ermittelte Salienz der Variante war also für die Zugezogenen, die aus Regionen außerhalb des Verbreitungsgebiets der Variante stammten, zweieinhalb bis dreimal so hoch wie für Personen, die in Ostwestfalen oder in anderen Regionen im Verbreitungsgebiet der Variante aufgewachsen sind. 
Die Auftragung der in der Untersuchung festgestellten, gemittelten Salienzwerte über der Aufenthaltsdauer der Zugezogenen in Ostwestfalen (Abb. 1) zeigt für die 32 Zugezogenen aus dem niederdeutschen Raum ein fast vollständiges Fehlen der Salienz der Variante. Für die Zugezogenen aus dem mittel- und oberdeutschen Raum dagegen ist die Variante salient. Mit steigender Aufenthaltsdauer steigen die Werte auf bis zu 50 Prozent an. Dieser Anstieg ist erstaunlich: Anstatt sich an die Regionalismen am neuen Wohnort zu gewöhnen, reagieren die Zugezogenen mit längerer Aufenthaltsdauer in Ostwestfalen zunehmend empfindlich darauf. Wie im Rahmen einer umfangreicheren Studie (cf. Nemeth 2011; Lorenz 2013; Lorenz i. V.) gezeigt werden konnte, geht dieser Anstieg der Salienzwerte einher mit der Vermeidung des Merkmals beim Vorlesen und Erzählen.

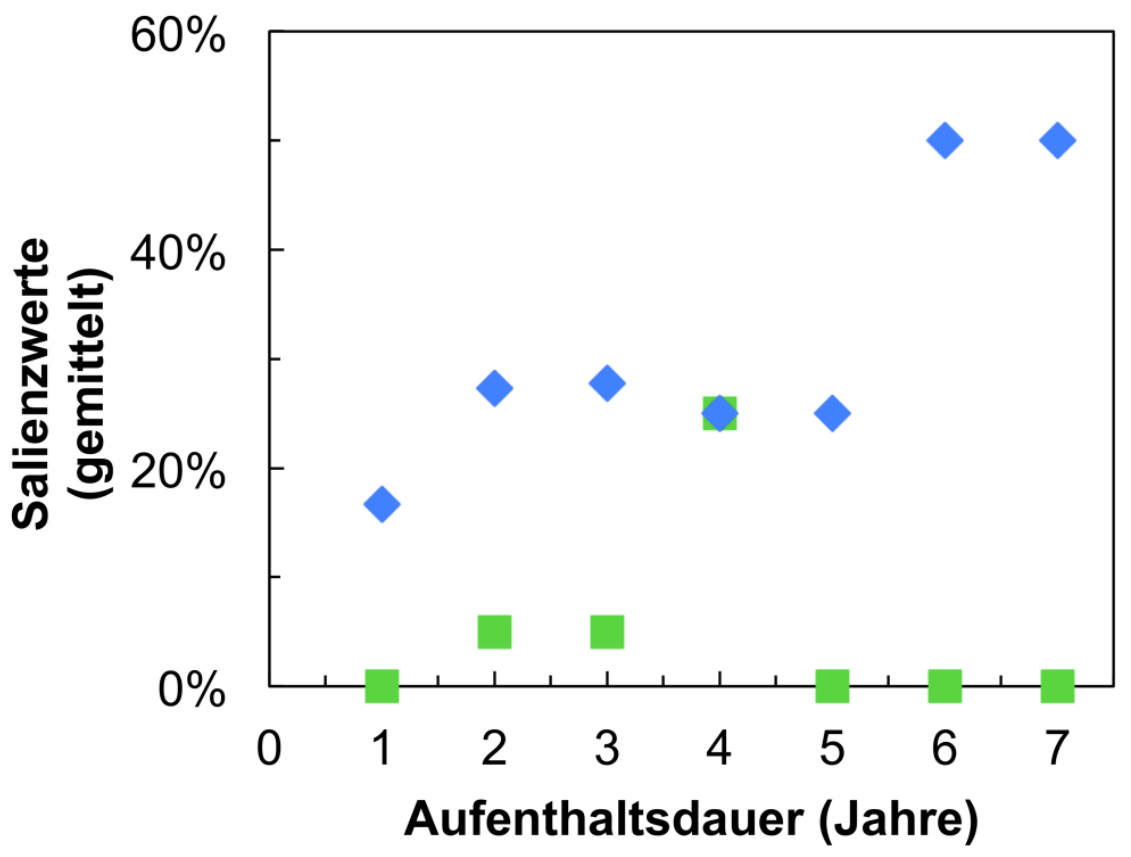

Abbildung 1: Salienzwerte der Realisierung von [ar] als Langmonophthong [a:] unter Zugezogenen aus dem niederdeutschen $(\square)$ und mittel- und oberdeutschen $(\diamond)$ Raum, aufgetragen über der Aufenthaltsdauer in Ostwestfalen.

\subsection{Sekundärdiphthongierung}

Die Salienz der Sekundärdiphthongierung wurde anhand des Stimulus W[o:a]t geprüft. Die Variante war für 25 Prozent aller Probanden salient.

Unter den einheimischen Ostwestfalen fiel der Mittelwert im Salienztest mit 14 Prozent niedrig aus. Unter den 32 Zugezogenen aus dem niederdeutschen Raum betrug er 19 Prozent und nach Ausschluss der sieben Personen aus dem autochthonen Verbreitungsgebiet der Variante 20 Prozent. Unter den mittel- und oberdeutschen Zugezogenen betrug der Mittelwert dagegen 44 Prozent; nach Ausschluss der beiden Personen aus dem Verbreitungsgebiet Regensburg (cf. König 1989b: 206-209; Lauf 1996: 206, 209; Mihm 2000: 2114, 2115) blieb dieser Wert konstant. Auch für diese Variante fällt die ermittelte Salienz unter den Zugezogenen außerhalb des Verbreitungsgebiets der Variante also zweieinhalb- bis dreimal so hoch aus wie für Personen, die aus dem Verbreitungsgebiet der Variante stammen.

Die Auftragung der gemittelten Salienzwerte über der Aufenthaltsdauer (Abb. 2) zeigt, dass die Werte unter den mittel- und oberdeutschen Zugezogenen mit der Aufenthaltsdauer stark ansteigen. Nach einer Aufenthaltsdauer von sechs Jahren erreichten die Salienzwerte sogar 100 Prozent. Auch für die Sekundärdiphthongierung konnte die umfangreichere Studie (cf. Nemeth 2011; Lorenz i. V.) nachweisen, dass die aktive Realisierung der Variante mit 
längerer Aufenthaltsdauer abnahm. Die Zugezogenen neigen also dazu, mit längerer Aufenthaltsdauer in Ostwestfalen die dort verbreiteten Regionalismen stärker wahrzunehmen und zu unterdrücken als nach kurzer Aufenthaltsdauer. Es handelt sich um eine Vermeidung. Bei den Zugezogenen aus dem niederdeutschen Raum ist eine gegenläufige Tendenz erkennbar: In dieser Gruppe sinken die ermittelten Werte mit zunehmendem Aufenthalt in Ostwestfalen (Abb. 2).

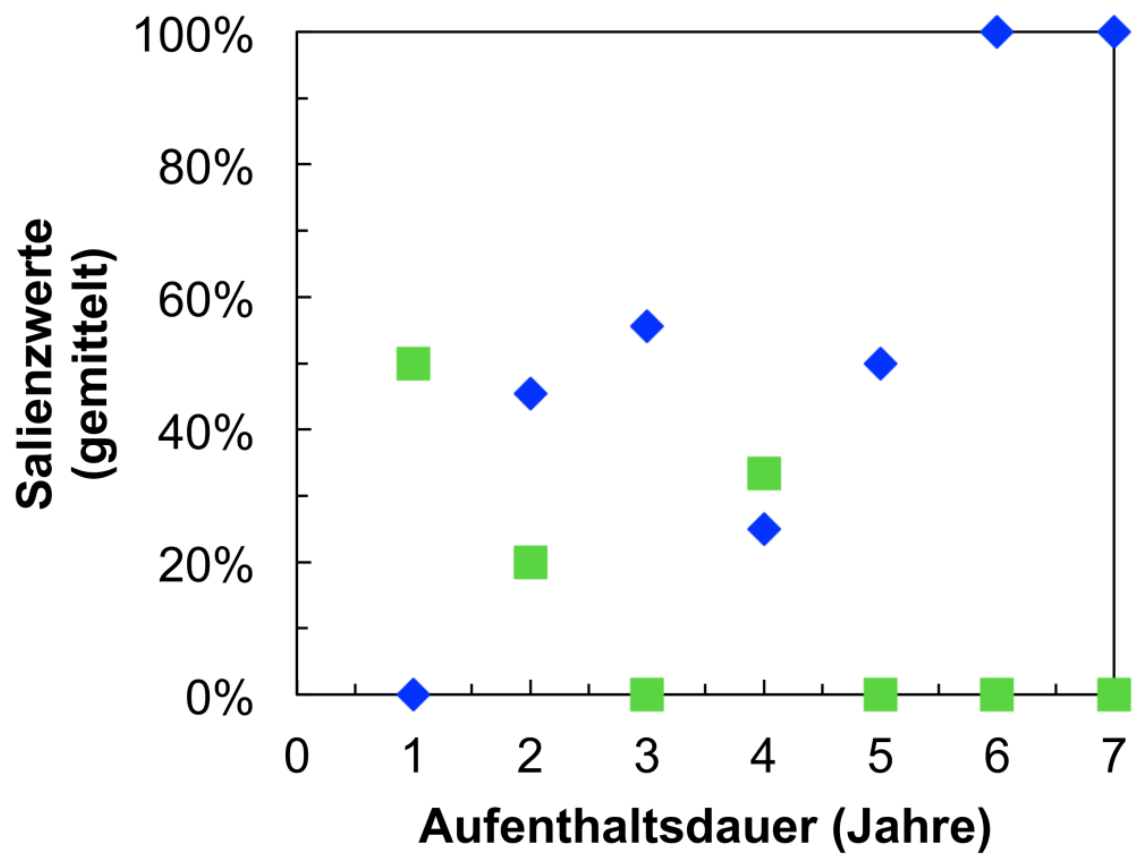

Abbildung 2: Salienzwerte der Sekundärdiphthongierung unter Zugezogenen aus dem niederdeutschen $(\square)$ und mittel- und oberdeutschen $(\diamond)$ Raum, aufgetragen über der Aufenthaltsdauer in Ostwestfalen.

\section{Zusammenfassung}

Die vorgestellte Studie über die Salienz unter einheimischen und zugezogenen jungen Erwachsenen in Ostwestfalen führte $\mathrm{zu}$ aussagekräftigen Ergebnissen: Die in der Untersuchung festgestellten, gemittelten Salienzwerte der vier regiolektalen Varianten unterschieden sich in Abhängigkeit der regionalen Herkunft der Probanden teils stark. Untersucht wurden die $g$-Spirantisierung im Auslaut nach Langvokal, die Realisierung von /pf/ im Anlaut als [pf] (standardnah) und [f] (regiolektal), die Realisierung von [ar] als Langmonophthong und die Sekundärdiphthongierung unter Einheimischen und Zugezogenen in Ostwestfalen.

Bei der $g$-Spirantisierung im Auslaut handelt es sich um ein hochsalientes Merkmal (cf. Elmentaler/Gessinger/Wirrer 2010: 120), das sowohl von Einheimischen als auch von Zugezogenen als auffällig wahrgenommen wurde: Fast jeder einzelne Proband gab beide Stimuli im Salienztest als auffällig an. Bei Merkmalen, die sich unter Einheimischen als hochsalient erweisen, müsste demnach nicht danach differenziert werden, ob die Salienzwerte unter Einheimischen oder Zugezogenen gemessen worden sind - die Merkmale sind unabhängig davon salient. Die regiolektale Realisierung der Affrikate /pf/ als [f] im Anlaut dagegen war ausschließlich für solche Zugezogene salient, die außerhalb der Verbreitungsregion des Merkmals aufgewachsen sind. Der gemittelte Salienzwert unter den einheimischen Ostwestfalen fiel nicht halb so hoch aus. Bei Varianten, die unter Einheimischen geringe Salienzwerte aufweisen (cf. Elmentaler/Gessinger/Wirrer 2010: 118) kann man also nicht davon ausgehen, dass die Variante generell als schwach salient 
einzuordnen ist. Vielmehr muss bei einer solchen Angabe unterschieden werden, ob die Variante unter Einheimischen oder Zugezogenen getestet wurde, denn je nach dem unterscheiden sich die Salienzwerte beträchtlich. Auch in Bezug auf die Realisierung von [ar] als Langmonophthong und die Sekundärdiphthongierung fielen die Salienzwerte zwischen Einheimischen und Zugezogenen unterschiedlich hoch aus: Die Salienzwerte unter den Ostwestfalen waren gering, die Werte unter solchen Zugezogenen, die nicht im Verbreitungsgebiet der Variante aufgewachsen sind, lagen deutlich höher. Es ist interessant, dass sich die Sensibilität der Zugezogenen gegenüber den Varianten mit der Zeit verändert: Sie steigt mit längerer Aufenthaltsdauer der Probanden in Ostwestfalen an. Bei solchen Merkmalen erscheint es sinnvoll, die Salienz nicht unter Einheimischen und Zugezogenen, sondern unter Einheimischen und Auswärtigen zu ermitteln, um möglichst konstante Salienzwerte zu erhalten.

Die Einheimischen in der vorliegenden Studie nahmen die $g$-Spirantisierung im Auslaut als einzige der in Ostwestfalen regiolektal verbreiteten Varianten als besonders auffällig wahr. Da sie sich also über die regionale Markiertheit dieser Variante bewusst sind, können sie dieses Merkmal bewusst verwenden (cf. Salewski 1998: 28; Reershemius 2009: 145) oder auch unterdrücken (cf. Elmentaler/Gessinger/Wirrer 2010: 120). Die in der Untersuchung festgestellten, gemittelten Salienzwerte der drei anderen in Ostwestfalen verbreiteten regiolektalen Merkmale fielen unter den Einheimischen weniger als halb so hoch aus wie bei der $g$-Spirantisierung im Auslaut. Daher ist davon auszugehen, dass die einheimischen Ostwestfalen die Verwendung dieser drei Merkmale nicht bewusst steuern können: Im Gegensatz zur g-Spirantisierung im Auslaut können sie solche Merkmale nicht gezielt zur Kennzeichnung einer informellen Situation oder ihrer regionalen Zugehörigkeit einsetzen. Für die Zugezogenen, die aus Regionen außerhalb der in Ostwestfalen verbreiteten regiolektalen Variante stammten, waren die $g$-Spirantisierung im Auslaut und die Realisierung der Affrikate /pf/ als [f] im Anlaut hochsalient. Im Gegensatz zu den Einheimischen nehmen die Zugezogenen also deutlich wahr, ob Einheimische diese Merkmale verwenden. Es bleibt aber offen, ob sie auch unterscheiden können, ob die Einheimischen solche Varianten bewusst oder unbewusst einsetzen. Die anderen beiden abgefragten Varianten wiesen unter den Einheimischen ausgesprochen niedrige und unter den Zugezogenen relativ niedrige Salienzwerte auf: Die Realisierung von [ar] als Langmonophthong und die Sekundärdiphthongierung fielen nur ungefähr einem Drittel der Zugezogenen auf, die nicht aus dem Verbreitungsgebiet der Variante stammten. Mit längerer Aufenthaltsdauer der Zugezogenen in Ostwestfalen erfolgte keine Angleichung an die niedrigen Salienzwerte der Einheimischen. Stattdessen stiegen die in der Untersuchung festgestellten, gemittelten Salienzwerte für beide Varianten unter den Zugezogenen mit längerer Aufenthaltsdauer stark an: Die Varianten, die den Einheimischen nicht bewusst sind, erschienen zunehmend auffälliger und wurden vermieden.

Der Vergleich der erhobenen Daten zeigt die gemessenen Salienzwerte und ihre zeitliche Entwicklung differenziert auf. Es wäre sicherlich sinnvoll, zu untersuchen, wie Zugezogene und Einheimische ihre Wahrnehmung der untersuchten regiolektalen Merkmale unmittelbar in Kommunikationssituationen einsetzen: Nehmen Einheimische und Zugezogene die untersuchten Merkmale auch außerhalb einer Testsituation als auffällig wahr? Unterscheiden sie den gezielten Einsatz eines regiolektalen Merkmals von der unbewussten Verwendung? Solche Fragen könnten in weiterführenden Einzelfallanalysen geklärt werden.

\section{Literatur}

Elmentaler, Michael (2005): "Die Rolle des überregionalen Sprachkontakts bei der Genese regionaler Umgangssprachen". Zeitschrift für deutsche Philologie 124: 395-415. 
Elmentaler, Michael et al. (2006): "Sprachvariation in Norddeutschland. Ein Projekt zur Analyse des sprachlichen Wandels in Norddeutschland". In: Gessinger, Joachim/Voeste, Anja (eds.): Dialekt im Wandel. Perspektiven einer neuen Dialektologie. Duisburg, Redaktion OBST: 159-178. (= Osnabrücker Beiträge zur Sprachtheorie 71).

Elmentaler, Michael/Gessinger, Joachim/Wirrer, Jan (2010): "Qualitative und quantitative Verfahren in der Ethnodialektologie am Beispiel von Salienz". In: Anders, Christina A./Hundt, Markus/Lasch, Alexander (eds.): Perceptual Dialectology. Neue Wege der Dialektologie. New York/Berlin, de Gruyter: 111-149. (= Linguistik - Impulse und Tendenzen 38).

Elspaß, Stephan/Möller, Robert (2003ff.): Atlas zur deutschen Alltagssprache. http://www.atlas-alltagssprache.de/, letzter Zugriff am 10.05.2013.

Hettler, Yvonne (2014): "Salienz, Bewertung und Realisierung regionaler Merkmale in Norddeutschland". Linguistik online 66: 71-90.

Hinskens, Frans/Auer, Peter/Kerswill, Paul (2004): "The Study of Dialect Convergence and Divergence. Conceptual and Methodological Considerations". In: Auer, Peter/Hinskens, Frans/Kerswill, Paul (eds.): Dialect Change. Convergence and Divergence in European Languages. Cambridge, Cambridge University Press: 1-48.

Hundt, Markus (2010): "Bericht über die Pilotstudie "Laienlinguistische Konzeptionen deutscher Dialekte". In: Anders, Christina A./Hundt, Markus/Lasch, Alexander (eds.): Perceptual Dialectology. Neue Wege der Dialektologie. New York/Berlin, de Gruyter: 179-220. (= Linguistik - Impulse und Tendenzen 38).

König, Werner (1989a): Atlas zur Aussprache des Schriftdeutschen in der Bundesrepublik Deutschland. Band 1: Text. Ismaning: Hueber.

König, Werner (1989b): Atlas zur Aussprache des Schriftdeutschen in der Bundesrepublik Deutschland. Band 2: Tabellen und Karten. Ismaning: Hueber.

König, Werner (2004): DTV-Atlas deutsche Sprache. 14., durchgesehene und aktualisierte Auflage. München: DTV.

Krech, Eva-Maria et al. (2009): Deutsches Aussprachewörterbuch. Berlin/New York: de Gruyter.

Lauf, Raphaela (1996): "'Regional markiert": Grossräumliche Umgangssprache(n) im niederdeutschen Raum". Niederdeutsches Jahrbuch. Jahrbuch des Vereins für niederdeutsche Sprachforschung 119: 193-218.

Lenz, Alexandra Nicole (2003): Struktur und Dynamik des Substandards. Eine Studie zum Westmitteldeutschen (Wittlich/Eifel). Stuttgart: Franz Steiner. $\quad(=$ Zeitschrift für Dialektologie und Linguistik. Beihefte 125).

Lenz, Alexandra Nicole (2010): "Zum Salienzbegriff und zum Nachweis salienter Merkmale". In: Anders, Christina A./Hundt, Markus/Lasch, Alexander (eds.): Perceptual Dialectology. Neue Wege der Dialektologie. New York/Berlin, de Gruyter: 89-110. (= Linguistik - Impulse und Tendenzen 38).

Lorenz, Cornelia (2013): "Übernahme von Regionalismen durch Zugezogene". In: Grucza, Franciszek et al. (eds.): Akten des XII. Internationalen Germanistenkongresses in Warschau 2010. Vielheit und Einheit der Germanistik weltweit. Bd. 17. Frankfurt a. M. etc., Peter Lang: 361-366. (=Publikationen der Internationalen Vereinigung für Germanistik (IVG) 17).

Lorenz, Cornelia (i. V.): Zugezogene im Fokus. Sprachkontakterscheinungen im Regiolekt. Universität Bielefeld. Dissertation, vom Promotionsausschuss angenommen 2013.

Mihm, Arend (1985): "Prestige und Stigma des Substandards. Zur Bewertung des Ruhrdeutschen im Ruhrgebiet". In: Mihm, Arend (ed.): Sprache an Rhein und Ruhr: dialektologische und soziolinguistische Studien zur sprachlichen Situation im Rhein-RuhrGebiet und ihrer Geschichte. Stuttgart, Franz Steiner: 163-194. (= Zeitschrift für Dialektologie und Linguistik Beihefte 50). 
Mihm, Arend (2000): "Die Rolle der Umgangssprache seit der Mitte des 20. Jahrhunderts". In: Besch, Werner et al. (eds.): Sprachgeschichte. Ein Handbuch zur Geschichte der deutschen Sprache und ihrer Erforschung. Band 2. 2., vollständig neu bearbeitete und erweiterte Auflage. Berlin/New York, de Gruyter: 2107-2137. (= Handbücher zur Sprachund Kommunikationswissenschaft 2.2).

Möller, Frerk (2008): Plattdeutsch im 21. Jahrhundert. Bestandsaufnahme und Perspektiven. Leer: Schuster. (= Schriften des Instituts für Niederdeutsche Sprache 34).

Nemeth, Cornelia (2011): "Zugezogene im Fokus. Eine empirische Studie". In: Ganswindt, Brigitte/Purschke, Christoph (eds.): Perspektiven der Variationslinguistik. Beiträge aus dem Forum Sprachvariation. Hildesheim etc., Olms: 99-120. (= Germanistische Linguistik 216/217).

Purschke, Christoph (2011): Regionalsprachlichkeit und Hörerurteil. Grundzüge einer perzeptiven Variationslinguistik. Stuttgart: Franz Steiner. (= Zeitschrift für Dialektologie und Linguistik Beihefte 149).

Reershemius, Gertrud (2009): "Post-Vernacular Language Use in a Low German Linguistic Community". Journal of Germanic Linguistics 21/2: 131-147.

Salewski, Kerstin (1998): Zur Homogenität des Substandards älterer Bergleute im Ruhrgebiet. Stuttgart: Franz Steiner. (= Zeitschrift für Dialektologie und Linguistik Beihefte 99).

Scheel, Käthe (1963): "Hamburger Missingsch". In: Simon, Werner/Bachofer, Wolfgang/Dittmann, Wolfgang (eds.): Festgabe für Ulrich Pretzel zum 65. Geburtstag, dargebracht von Freunden und Schülern. Berlin, Erich Schmidt: 381-389.

Schirmunski, Viktor M. (1928/1929): "Die schwäbischen Mundarten in Transkaukasien und Südukraine". Teuthonista 5: 38-60, 157-171.

Schmidt, Jürgen Erich/Herrgen, Joachim (2011): Sprachdynamik. Eine Einführung in die moderne Regionalsprachenforschung. Berlin: Erich Schmidt. (= Grundlagen der Germanistik 49).

Stellmacher, Dieter (1977): Studien zur gesprochenen Sprache in Niedersachsen. Eine soziolinguistische Untersuchung. Marburg: Elwert. (= Deutsche Dialektgeographie 82).

Stellmacher, Dieter (1987): Wer spricht Platt? Zur Lage des Niederdeutschen heute. Eine kurzgefaßte Bestandsaufnahme. Leer: Schuster. (= Schriften des Instituts für Niederdeutsche Sprache 14).

Stellmacher, Dieter (2000): Niederdeutsche Sprache. 2., überarbeitete Auflage. Berlin: Weidler. (= Germanistische Lehrbuchsammlung 26).

Trudgill, Peter (1986): Dialects in Contact. Oxford: Basil Blackwell. (= Language in Society $10)$.

Wirrer, Jan (1998): "Zum Status des Niederdeutschen". Zeitschrift für germanistische Linguistik 26: 308-340. 